\title{
Plastid Ultrastructure in Chlorophyll Mutant Sectors of Leaves in Seed-Irradiated Lactuceae
}

\author{
M. Z. Haque and M. B. E. Godward \\ School of Biological Sciences \\ Queen Mary College (University of London), \\ Mile End Road, London E1 4NS, U.K.
}

Accepted August 28, 1985

Seed-irradiated Lactuceae produced an $\mathrm{M}_{1}$ generation which included plants with leaf-sectors which were mutant for chlorophyll characters. These were examined with the electron microscope with a view to comparing their ultrastructure with that of barley chlorophyll mutants well known from the detailed researches of Diter von Wettstein (1980) and his collaborators at the Carlsberg Laboratories in Copenhagen.

\section{Materials and methods}

The plants used were Lactuca sativa $\mathrm{L}$. cultivars Tom Thumb, Webb's Wonderful and Lobjoit's Green Cos grown from seeds obtained from Samuel Dobie and Sons Ltd., Llangollen, Clwyd, North Wales (U.K.) and Lactuca serriola L., collected from the wild in the vicinity of Waltham Abbey, Essex, U.K. and kindly provided by Dr. R. N. Carter of the School of Biological Sciences, Queen Mary College.

In order to have seeds of the same age and uniformity the original seeds were sown and grown up in greenhouses under standard conditions which were uniform but prevented any cross-pollination; the resulting seeds of these plants were those used in the investigation.

Following desiccation over calcium chloride $\left(\mathrm{CaCl}_{2}\right)$ till no further loss of weight occurred, seven samples each of 600 dormant seeds from each cultivar and L. serriola L. were irradiated with a dose range of ten to eighty KR of Gamma rays from a ${ }^{80} \mathrm{Co}$ source at Queen Mary College delivering $3500 \mathrm{R} / \mathrm{min}$.

\section{Method of cultivation}

Each control or irradiated sample of 250 seeds was sown in seed boxes, in the roof greenhouse at QMC and at the Field Station at Dytchleys in Essex. They were equally spaced out 50 to a seed box $(350 \times 250 \mathrm{~mm})$ at $17^{\circ}$ to $22^{\circ} \mathrm{C}$, with additional light from $6.0 \mathrm{a} . \mathrm{m}$. to $6.0 \mathrm{p} . \mathrm{m}$. using 500 Watt mercury vapour lamps type $\mathrm{MBU}$, in autumn and winter.

Five weeks after sowing, all the surviving seedlings were transplanted into the field in randomised arrangements as provided by Fisher and Yates (Fisher and Yates 1953).

At QMC 15 seedlings ( 20 in L. serriola) from each treatment i.e. controls and at 
each dose, of all the cultivars and $L$. serriola were taken at random and transplanted into 6" plastic pots filled with sterilized John Innes Compost No. 2. The pots were randomized in the greenhouses and as far as possible similar conditions were provided to all the plants.

When the $M_{1}$ and $M_{2}$ plants of Lactuca cultivars and species were about eight weeks old, samples of leaf sectors not longer than $1 \times 3 \mathrm{~mm}$ were carefully cut from controls (leaves of non-irradiated plants), from the normal green parts of sectorial chimaeric leaves from irradiated seeds, and from the pale yellowish-white sectors.

The cut materials were fixed in $4 \%$ glutaraldehyde in sodium cacodylate buffer $\mathrm{pH}=7.0$ for two hours at room temperature, washed in buffer, post-fixed in $1 \%$ $\mathrm{OsO}_{4}$ (osmic acid) for two hours, and washed again in buffer before dehydration in an alcohol series followed by propylene oxide.

Infiltration and embedding was in TAAB resin. Sectioning was done on LKB III and Cambridge Huxley microtomes with glass knives; sections mounted on 200 mesh, uncoated copper grids, and stained with $2 \%$ alcoholic uranyl acetate followed by lead citrate (Reynolds 1963).

The sections were examined and photographed on a Zeiss E. M. 9 operating on $60 \mathrm{KV}$ and Siemens Elmiskop IA.

\section{Observations}

The ultrastructure of the control materials of the cultivars and species studied, shows no pronounced differences between them. A very small amount of cytoplasm lies between the plasma-membrane and the plastid on the one side and on the other between the plastid and the tonoplast (Fig. 1).

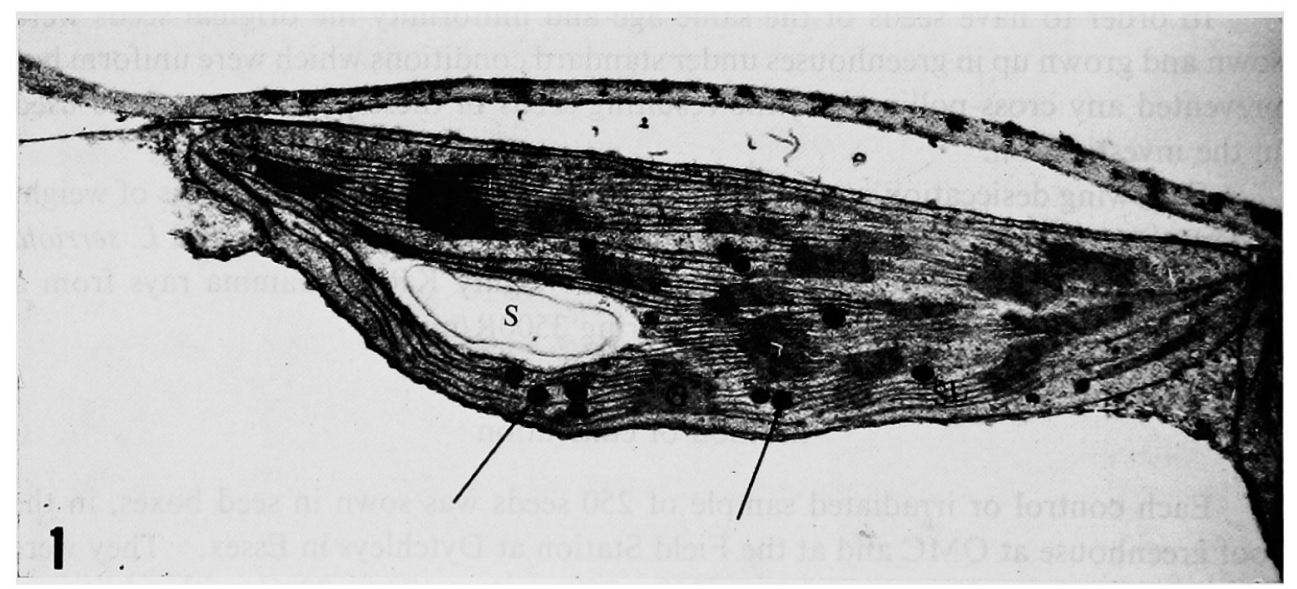

Fig. 1. Lactuca sativa cultivar Cos, typical control material, plastid showing grana (G) and stroma lamellae (S.L.), one starch grain (S), and a few lipid droplets $(\rightarrow) . \quad \times 17300$.

Nearly every thylakoid of a granum is continued into the stroma as a stroma lamella. The number of stroma lamellae visible in the sections varies from 2-11. The number of grana visible in a section of plastid is $10-16$, and the number of thylakoids stacked in a granum, 3-17. 1-3 starch grains and 3-13 lipid globules 

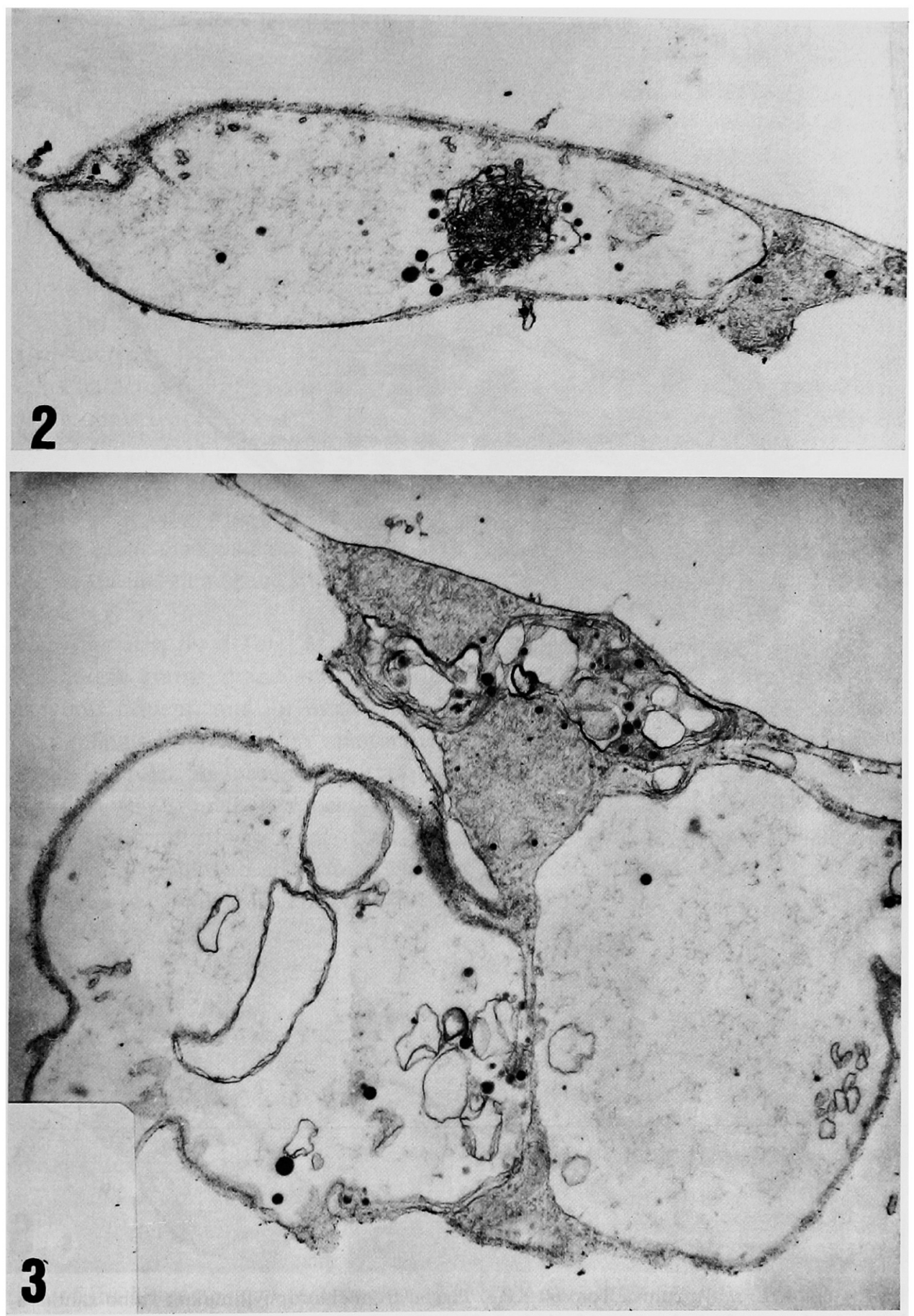

Figs. 2-3. 2, L. sativa cultivar Cos, $\mathrm{M}_{1}$ plant, $30 \mathrm{KR}$. Plastid from chlorophyll mutant "alboxantha $a_{1}$ ". Irregular mass of tubules and membranes reminiscent of a prolamellar body in distorted form. No normal lamellae are present. Lipid droplets occur near the body. $\times 21000$. 3, $L$. sativa cultivar Cos, $M_{1}$ plant, $30 \mathrm{KR}$. Plastid from chlorophyll mutant "albo-xantha $a_{1}$ ". Only a few empty vesicles and small lipid droplets are present. $\quad \times 23300$. 

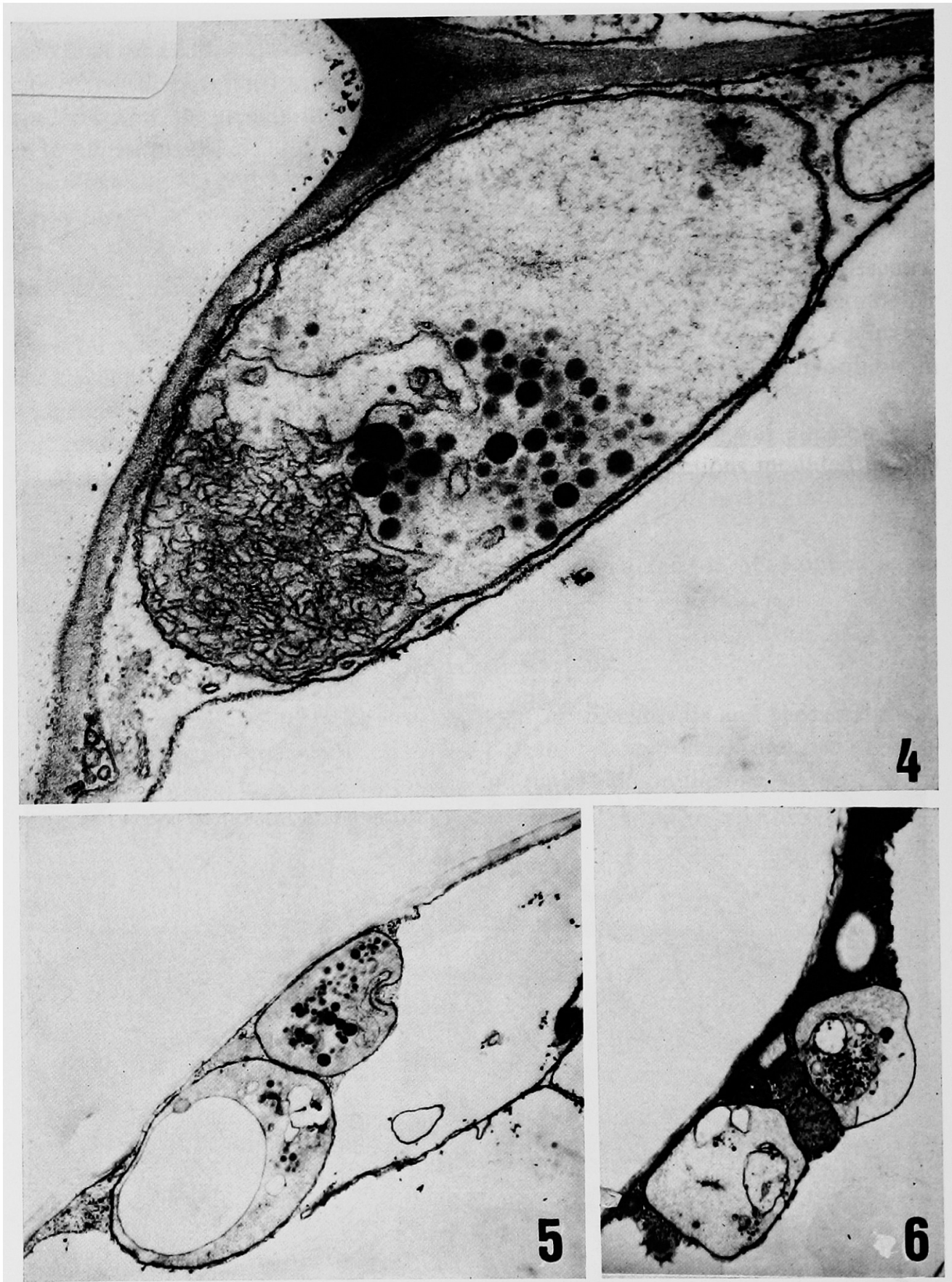

Figs. 4-6. 4, L. sativa cultiva Tom $60 \mathrm{KR}$. Plastid from chlorophyll mutant "albo-xantha $\mathrm{a}_{2}$ ", intertwined mass of membranes and tubules. Numerous lipid droplets occur near them. $\times 24000$. 5, $\times 4950$. 6, L. sativa cultivar Tom, $M_{1}$ plant, $60 \mathrm{KR}$. Plastid from "albo-xantha $a_{2}$ ", showing only a few distorted membranes and vesicles, with associated lipid droplets. $\times 14250$. 
are also visible in the stroma of the plastids.

Two mutant plastid types are clearly distinguishable, both from yellow-white sectors of leaves. Although these are not necessarily identical with mutants observed and identified in barley (von Wettstein $e t$ al. 1971) they are conveniently described by provisional terms similar to those used for barley and in a general way for other material. These plastid types are referred to below as albo-xantha ' $a$ ' and alboxantha ' $b$ '.

\section{i. Albo-xantha' ' $a$ '}

There are two categories, $a_{1}$ and $a_{2}$. Albo-xantha $a_{1}$ from Cos, $M_{1}$

Characteristic of this are the following features: Grana, stroma lamellae and starch grains are absent. A mass of intertwined tubules associated with lipid globules is present in the centre of the plastid. Small regions of this mass are faintly reminiscent of a prolamellar body. A few scattered irregular membranous structures occur in the otherwise empty stroma (Fig. 2).

In other plastids from the same sector, there was no development of the intertwined tubule-membrane stucture, and only a few empty vesicles and scattered lipid droplets (Fig. 3).

Albo-xantha $a_{2}$ from Tom, $M_{1}$

Starch grains, grana and stroma lamellae are absent, lipid globules of various sizes are present, and an intertwined mass of membranes and tubules associated with a single large irregular membrane is present at one end of the plastid (Fig. 4). Small irregular membranous structures are also present. The plastid shown in Fig. 6 is very similar in appearance to that in Fig. 2 of Cos. However, more extreme developments in Tom include reduction of the intertwined mass of membranes and tubules to a few remenants (Figs. 5,6) and elimination of all but a few large or small vesicular bodies and all but a few lipid droplets (Fig. 6). The colour was paler.

\section{ii. Albo-xantha ' $b$ '}

Again there are two categories, $b_{1}$ and $b_{2}$. Albo-xantha $b_{1}$

The $M_{1}$ material, from $L$. serriola, is illustrated in Figs. 7 and 8 .

In the sections, it can be seen that grana are missing but limited numbers of stroma lamella are present, many of them vesiculated but still aligned with the long axis of the plastid (Figs. 7,8).

No starch grains are found, but there are limited numbers of lipid droplets. Occasional larger vesicles may appear (Fig. 7). In more extreme instances, vesiculation of the lamellae is more pronounced (Fig. 8), and there are more numerous lipid droplets.

Albo-xantha $b_{2}$

This $M_{2}$ mutant is from Webbs. The same clearly defined features, alignment of vesiculated stroma lamellae, not too numerous lipid droplets, and an occasional large vesicles, are found. Here however the alignment is more marked, and there 


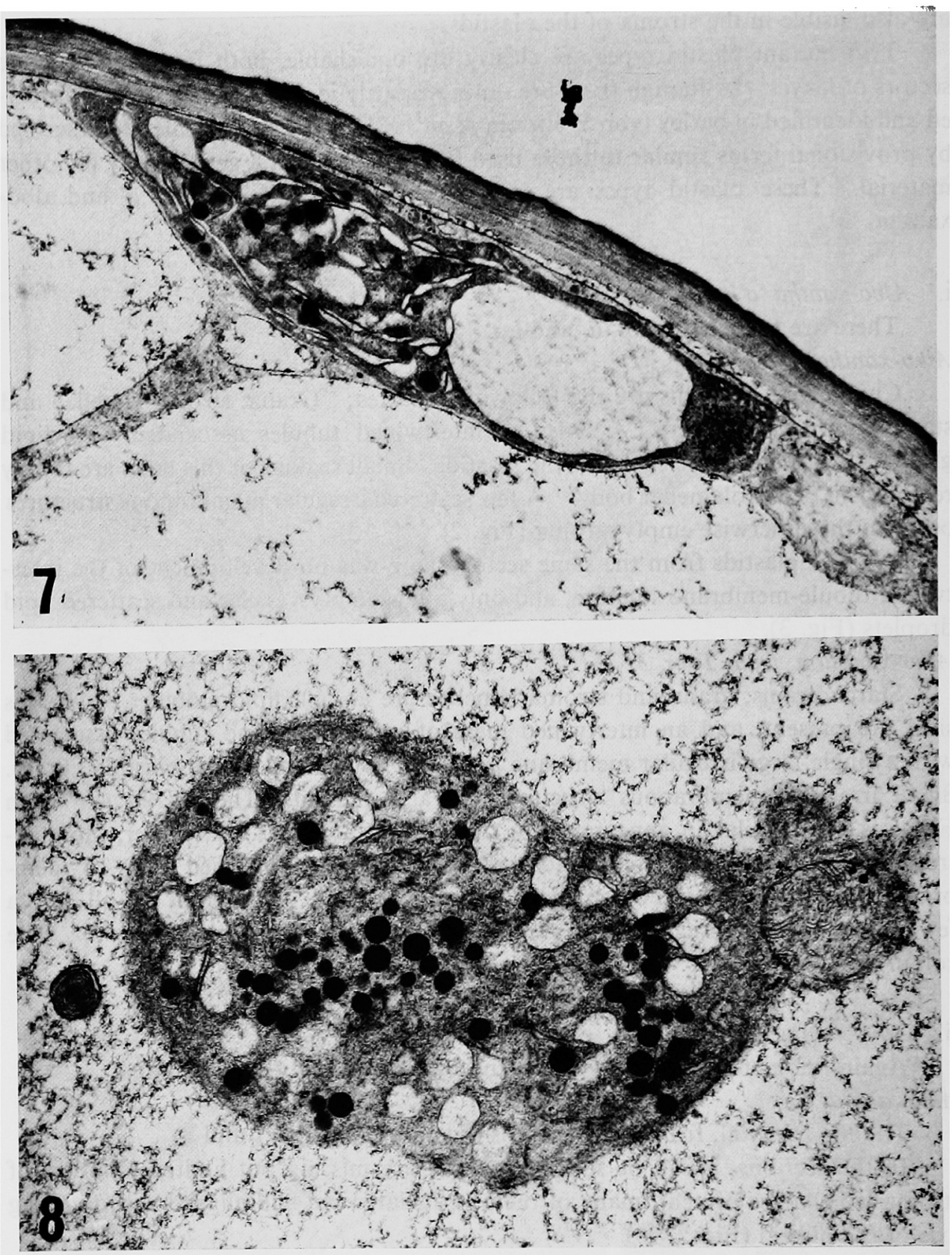

Figs. 7-8. 7, L. serriolia, $M_{1}$ plant, $30 \mathrm{KR}$, "albo-xantha $b_{1}$ " chlorophyll mutant. No grana lamellae are present, but stroma lamellae, many of them ulvesicated, are more or less aligned with the long axis of the plastid. A large vesicle and limited number of lipid droplets are present. $\times 20080$. 8, L. serriola, $M_{1}$ plant, $30 \mathrm{KR}$, "albo-xantha $b_{1}$ " chlorophyll mutant. No grana lamellae are present, but stroma lamellae, many of them vesiculated, are more or less aligned with the long axis of the plastid. Numerous lipid droplets present. $\quad \times 21090$. 

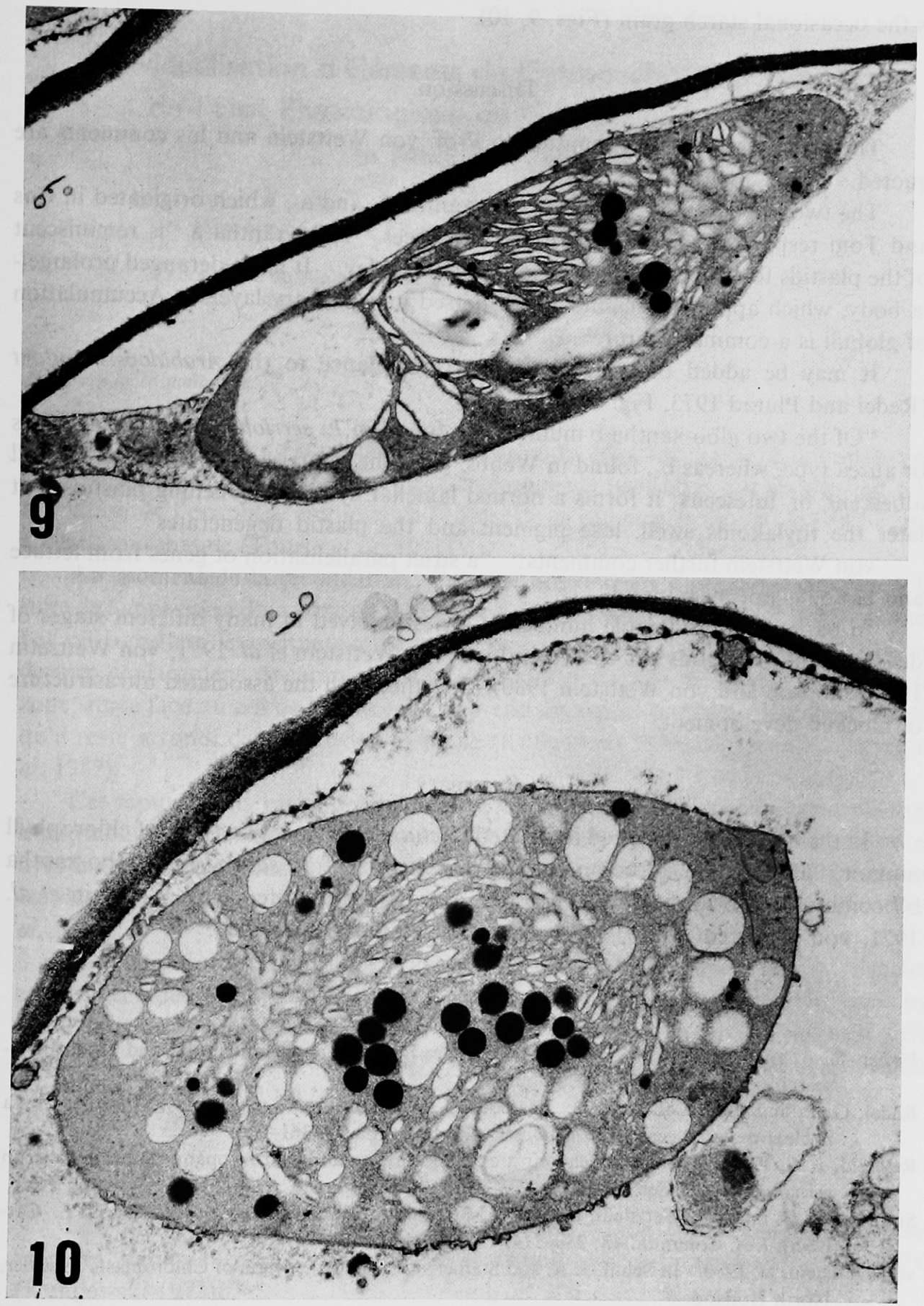

Figs. 9-10. 9, L. sativa cultivar Webbs, $\mathrm{M}_{1}$ plant, $30 \mathrm{KR}$. "albo-xantha $\mathrm{b}_{2}$ " chlorophyll mutant. Vesiculated and mostly aligned stroma lamellae, no grana, few lipid droplets, occasionaly large vesicle, and starch grains are present. $\quad \times 16000.10$, L. sativa cultivar Webbs, $M_{1}$ plant, $30 \mathrm{KR}$. "albo-xantha $b_{2}$ " chlorophyll mutant. Vesiculated and mostly aligned stroma lamellae, no grana, numerous lipid droplets, occasionaly large vesicle and a small starch grain are present. $\times 20000$. 
is the occasional starch grain (Figs. 9, 10).

\section{Discussion}

The micrographs were submitted to Prof. von Wettstein and his comments are quoted.

The two 'albo-xantha' mutants, albo-xantha $a_{1}$ and $a_{2}$, which originated in Cos and Tom respectively show marked resemblances. Albo-xantha a "is reminiscent of the plastids found in the mutant Xantha-u of barley. It has a deranged prolamella body, which apparently cannot be dispersed into primary layers. Accumulation of globuli is a common feature".

It may be added that there is some resemblance to the Arabidopsis mutant (Redei and Plurad 1973, Fig. 6d).

"Of the two albo-xantha b mutants, $\mathrm{b}_{1}$, found in $L$. serriola, looks like a viridis or aurea type, whereas $b_{2}$, found in Webbs, gives the impression of the type we call albescens or lutescens; it forms a normal lamellar system in greening plastids, but later the thylakoids swell, lose pigment and the plastid degenerates".

von Wettstein further comments: "a strict parallelisation of genes from lettuce and barley is not meaningful".

In barley, a considerable number of genes involved in many different stages of development of plastids has been identified (von Wettstein et al. 1971, von Wettsetin 1980, Simpson and von Wettstein 1980)—-together with the associated ultrastructure of blocked development.

\section{Summary}

In the $\mathrm{M}_{1}$ plants from seed irrdaiated Lactuceae, at least two types of chlorophyll mutant, "albo-xantha a" common to two cultivars of $L$. sativa, and "albo-xantha b" common to $L$. sativa and $L$. serriola, were recognised (c.f. von Wettstein $e$ t al. 1971, von Wettstein 1980). They are illustrated and discussed.

\section{References}

Fisher, R. A. and Yates, F. 1953. Statistical Tables for Biological Agricultural and Medical Research. Oliver and Boyd, Edinburgh.

Redei, G. P. and Plurad, S. B. 1973. Hereditary structural alterations of plastids induced by a nuclear mutator gene in Arabidopsis. Protoplasma 77: 361-380.

Reynold, E. S. 1963. The use of lead citrate at high pH as an electron opaque stain in electron microscopy. J, Cell Biol. 17: 208-212.

Simpson, D. J. and von Wettstein, D. 1980. Macromolecular physiology of plastids XIV. Carlsberg Res. Commun. 45: 283-314.

von Wettstein, D. 1980. In Schiff. J. A. and Stainer, R. Y. (Eds.), Origin of Chloroplasts Elsevier, North Holland.

-, Henningsen, J. E., Boynton, G. C., Kannangara and Neilson, O. 1971. The genic control of chloroplast development in barley. pp. 205-223. In: Autonomy and Biogenesis of Mitochondria and Chloroplasts. (Eds. N. K. Boardman, A. W. Linnane and R. M. Smillie). North Holland, Amsterdam. 